\title{
Stellar contents of the open clusters Be 64 and Be $69^{\star}$
}

\author{
A.K. Pandey ${ }^{1}$, A.K. Durgapal ${ }^{1}$, B.C. Bhatt ${ }^{2}$, V. Mohan ${ }^{1}$, and H.S. Mahra ${ }^{1}$ \\ 1 Uttar Pradesh State Observatory, Manora peak, Naini Tal, 263129, India \\ 2 Indian Institute of Astrophysics, Koramangala, Bangalore, 560034, India
}

Received January 12; accepted July 3, 1996

\begin{abstract}
We present $U B V R I$ CCD photometry for previously unstudied open clusters (Be 64 and $\mathrm{Be} 69$ ). Photometry has also been carried out for nearby fields to correct for the effects of field stars contamination. From the colour-colour diagram the reddening for Be 64 and Be 69 is estimated to be $\sim 1.05 \mathrm{mag}$ and $\sim 0.65 \mathrm{mag}$ respectively. In case of $\mathrm{Be} 69$ the comparison of observational colour - magnitude diagram (CMD) with the standard isochrones of VandenBerg (1985) indicates an apparent discrepancy between the shape of the turnoff and isochrones. The morphological features of the CMDs of $\mathrm{Be} 69$ are better understood in terms of convective overshooting. The comparison of CMDs of both the clusters with the convective overshoot models (Bertelli et al. 1994) produces a good fit for a metallicity $Z=0.008$ and age $=0.8-1.0$ Gyr. An apparent distance modulus $(m-M)=16.2$ and 14.3 has been estimated for Be 64 and $\mathrm{Be} 69$, respectively, which corresponds to a distance of $3880 \pm 480 \mathrm{pc}$ and $2860 \pm 310 \mathrm{pc}$, respectively.
\end{abstract}

Key words: open clusters: Be 64; Be 69 - HR diagram

\section{Introduction}

Star clusters constitute a principal link between the theories of stellar evolution and the observable universe. Open clusters are important tools to analyze the properties of the galactic disk and to test the theories of galactic and stellar evolution. The comparison between the HR diagram of the observed clusters and the isochrones, computed by means of evolutionary models, provides a test of stellar structure and evolution. The comparison provides an opportunity to ascertain whether or not convective overshoot plays an important role in the evolution.

Only about one third of the open clusters of the Milky Way have well studied HR diagrams to estimate

Send offprint requests to: A.K. Pandey

* Tables 2 and 3 are available in electronic form only at the CDS via anonymous ftp 130.79.128.5.

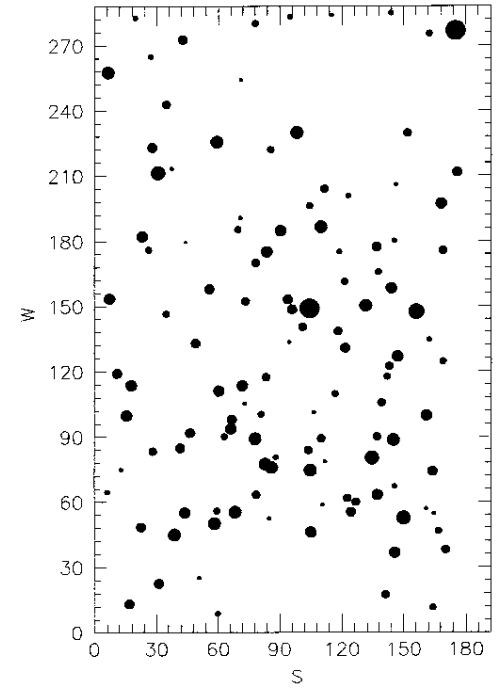

Fig. 1. Identification map for the cluster Be 64
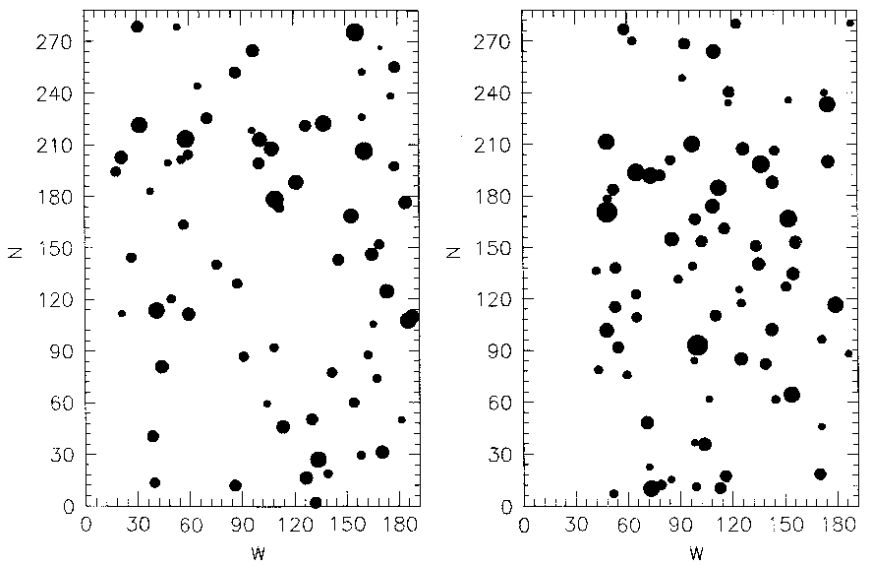

Fig. 2. Identification map for the cluster Be 69 

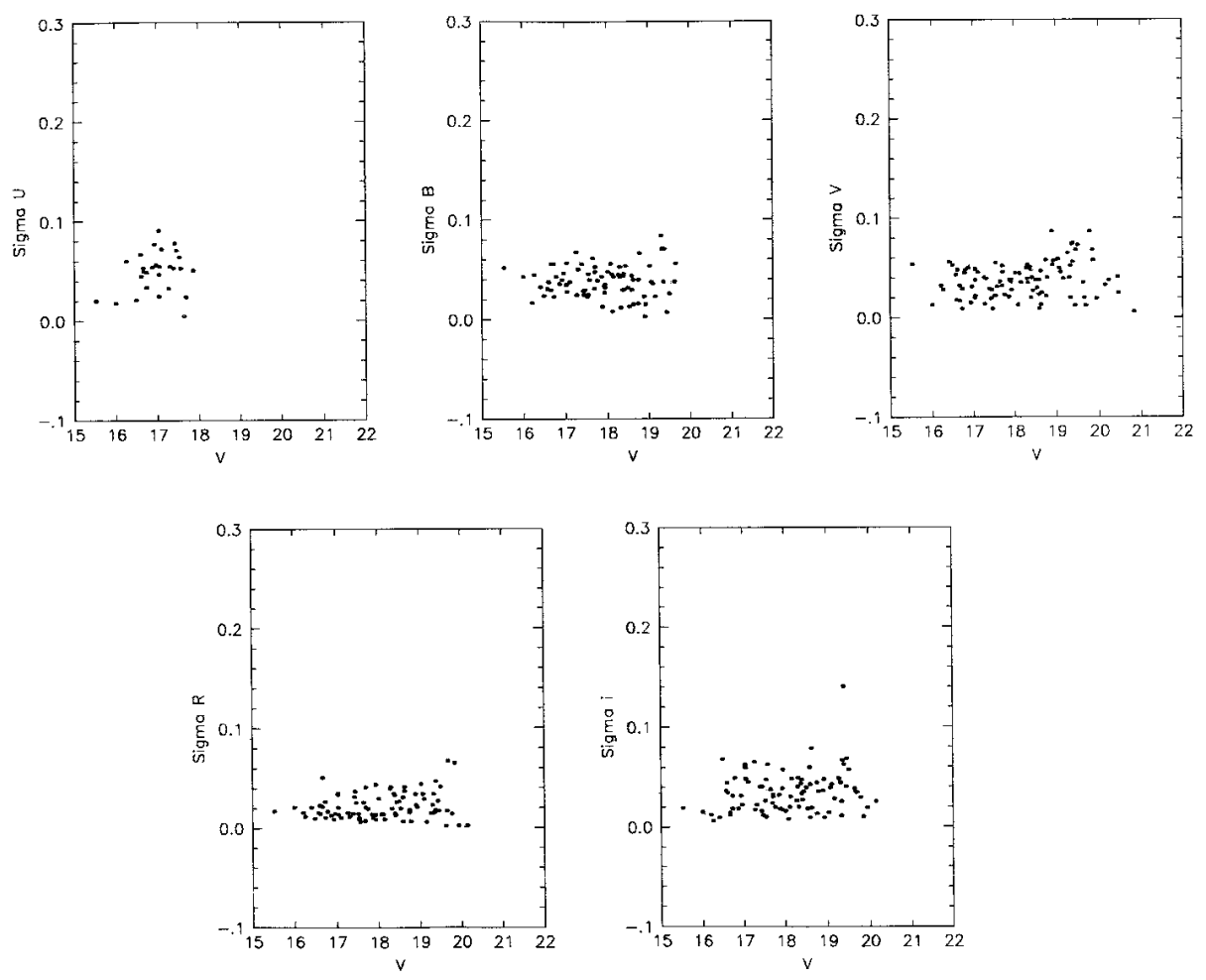

Fig. 3. The internal errors as a function of $V$ magnitude

their ages and other parameters and most of them are much younger than the galaxy. Most of the open clusters are destroyed by interaction with molecular clouds on time scales of a few hundred million of years or less (Spitzer 1958). Janes \& Adler (1982) and Pandey et al. (1987) found that the median age of clusters is about $10^{8}$ yrs. However, some open clusters survive for billions of year and few may be as old as the disk.

There is currently much interest shown by various groups to determine the accurate age for intermediate age and old open clusters (see e.g., Kaluzny 1990, 1994; Janes \& Phelps 1990; Twarog et al. 1993; Phelps et al. 1994; Carraro \& Chiosi 1994) because the determination of age of clusters would allow us to set a lower limit to the age of the galactic disk. The understanding of their properties (age, metallicity and kinematics) is mandatory for many studies, such as the history of star formation in the galactic disk, the formation and structure of the disk.

With the aim to contribute to the progress in our understanding of this population we have undertaken an observational program to obtain reliable $U B V R I$ CCD photometry of those clusters which are unstudied or poorly studied.

In this paper, we present CCD $U B V R I$ photometry for the open clusters Be $64(\mathrm{C} 0217+656, l=131.9, b=4.6)$ and Be $69(\mathrm{C} 0521+326, l=174.4, b=-1.8)$, for which no previous photometric studies could be found in the literature and compare their colour - magnitude diagrams (CMDs) with the theoretical ones.

\section{Observations and reductions}

The UBVRI CCD photometric observations were obtained, using photometric CCD system at $\mathrm{f} / 13$ cassegrain focus of the 104-cm telescope of the Uttar Pradesh State Observatory (UPSO) on nights during October December 1990 and November - December 1992. In order to improve the $\mathrm{S} / \mathrm{N}$ ratio, the observations were taken in binning mode of $2 \times 2$ pixels. In this setup, each pixel of $384 \times 576$ size CCD corresponds to 0.66 arcsec and the entire chip covers a field of $2.0 \times 3.0$ arcmin. The details of the CCD system have been given in Mohan et al. (1991).

In case of Be 64 one field centred on the cluster was observed (Fig. 1). In addition to it, two fields situated $\approx 30^{\prime}$ towards east and west of the cluster were also observed. For Be 69 two overlapping fields called north and south were imaged to cover the cluster region (Fig. 2). Also, two nearby fields towards north and south of the cluster situated $30^{\prime}$ away from the cluster centre were observed. Multiple exposures with exposure times ranging from $10 \mathrm{~s}$ to $900 \mathrm{~s}$ depending on the presence of bright stars and filter used were taken. Details of observations have been given in Table 1. We have sandwiched the exposures of each field in each filter with exposure of a comparison field in the same filter. The comparison frames were in the vicinity of the 
cluster regions. The observations were carried out within two hours of meridian so that air mass change within a given sandwich was only marginal. The comparison fields were standardised using Landolt's (1983) stars. A number of bias and twilight flat - field frames were also taken on several nights during the observing runs.

The data analysis was carried out using the computing facilities (Vax Station and Alpha Station) available at the Observatory. The frames were cleaned employing the standard procedures using ESO MIDAS softwares running on the above systems of the Observatory. Different clean frames of same field in the same filter were coadded. Photometry of co-added frames was carried out using DAOPHOT photometric package by Stetson (1987). PSF was obtained for each frame using several uncontaminated stars and the PSF magnitudes were suitably tied to aperture photometry magnitudes. For comparison fields, standard stars and bright stars in the cluster fields, only aperture photometry was obtained.
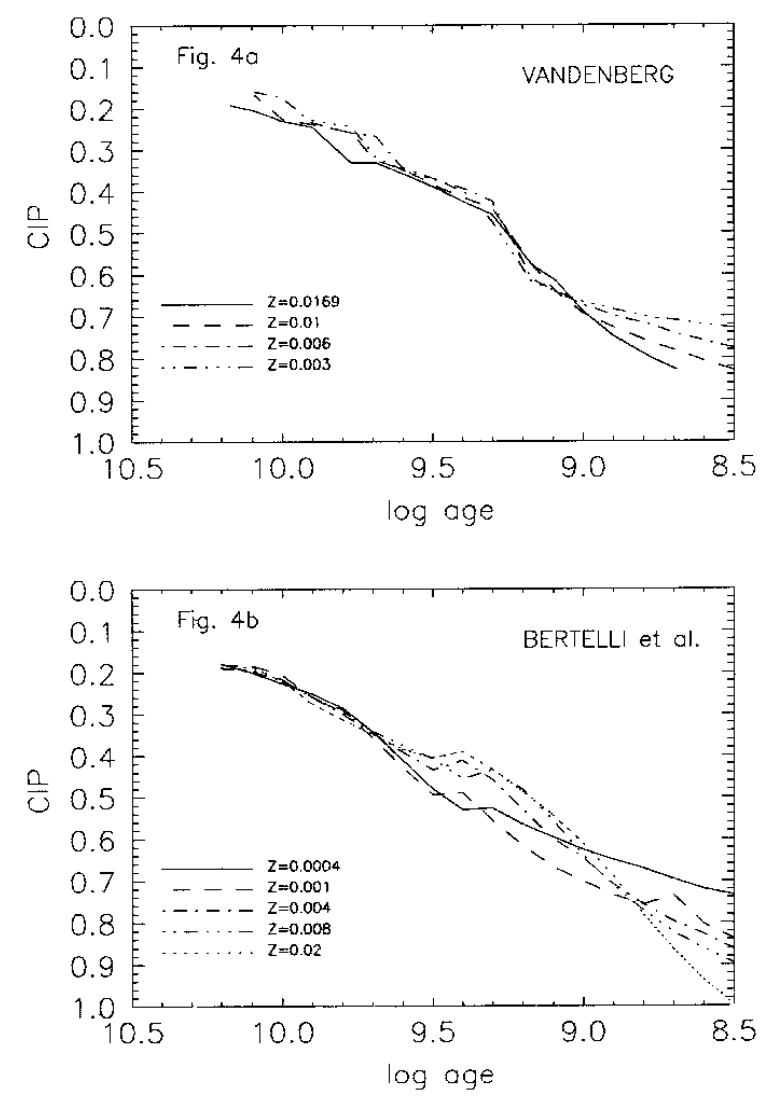

Fig. 4. a) The variation of colour - index parameter (CIP) with age. The data have been taken from Vandenberg (1985). b) Same as Fig. 4a. The data have been taken from Bertelli et al. (1994)

For each frame, differential magnitudes and colours were obtained using the observed magnitudes of the comparison field. There differential magnitudes were then standardised using the transformation equations obtained by Mohan et al. (1991) for the CCD system.

$\Delta(B-V)=1.192 \Delta(b-v) \pm 0.007$

$\Delta(U-B)=0.918 \Delta(u-b) \pm 0.008$

$\Delta(V-R)=0.898 \Delta(v-r) \pm 0.007$

$\Delta(R-I)=1.016 \Delta(r-i) \pm 0.008$.

For $V$ magnitudes no colour term could be determined. The internal errors estimated from the scatter in the individual measures of different exposures are shown in Fig. 3 as a function of $V$ magnitude. The average value of $\sigma$ for stars having $V \leq 18.0$ mag comes out to be $\sigma_{u}=0.05, \sigma_{b}=0.04$, $\sigma_{v}=0.03, \sigma_{r}=0.02$ and $\sigma_{i}=0.03$ whereas, for stars having $V>18.0 \mathrm{mag}, \sigma_{b}, \sigma_{v}, \sigma_{r}, \sigma_{i}$ comes out to be 0.04 . The $X$ and $Y$ co-ordinates as well as photometric data of the stars measured in the clusters Be 64 and Be 69 have been given in Tables 2 and 3 respectively.

\section{Morphological age of the clusters}

Age of an open cluster is obtained by fitting theoretical isochrones to its colour - magnitude diagrams. This requires however, a knowledge of cluster metallicity and reddening. Distance modulus can be determined simultaneously with age. The difficulties encountered in fitting theoretical isochrones to observed cluster CMDs when no information about reddening and/or metallicity is available, have prompted some indirect methods for age estimation of open clusters. These methods use the visible differences in the CMDs of star clusters for different ages (see e.g., Kaluzny 1994; Phelps et al. 1994), the position of red giant clump, main sequence blue turnoff (BTO) and red turnoff point.

We have used another colour index parameter (CIP) which is the difference in colour index between the blue turnoff (BTO) point of the main sequence and the colour at the base of the red giant branch (BRGB). This parameter can be useful in clusters having no noticeable clump. This parameter slightly depends on metallicity but still can be used to have an estimate of age of intermediate age clusters. The variation of the colour index parameter with age has been shown in Figs. 4a and 4b. The data have been taken from VandenBerg (1985) and Bertelli et al. (1994).

\section{Colour-magnitude diagrams (CMDs)}

\subsection{Be 64}

The CMDs for Be 64 are shown in Fig. 5. Two nearby fields towards east and west directions $30^{\prime}$ away from the cluster region have also been observed to estimate contamination of field stars in the cluster region. The CMDs for field regions are shown in Fig. 6. A clump of red giants appears near $V=16.4,(B-V) \sim 1.8,(V-I) \sim 2.2$, $(V-R) \sim 1.1$. A comparison of the $(V, V-R)$ and 

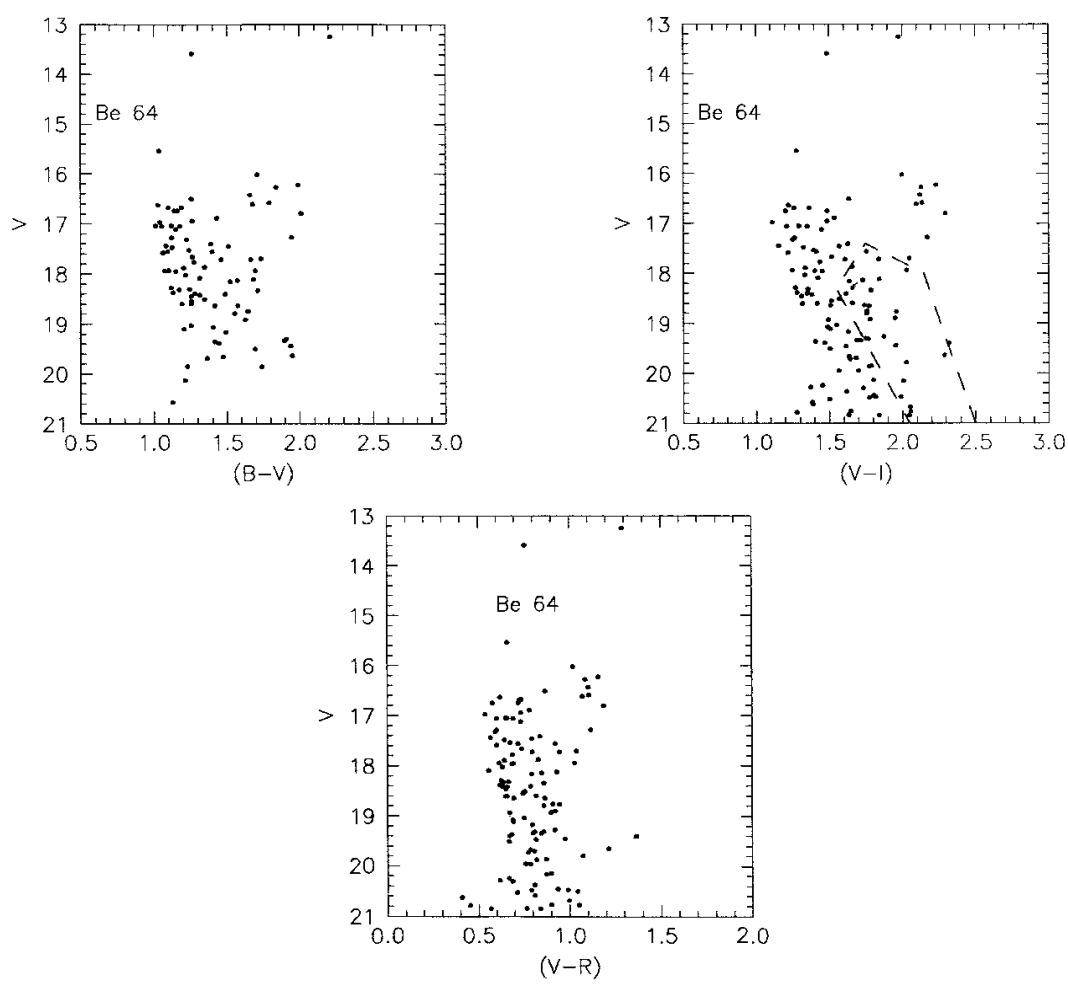

Fig. 5. The CMDs for all stars in the Be 64 region. The stars lying in the box in $(V, V-I)$ CMD mainly belong to the foreground population (see text)
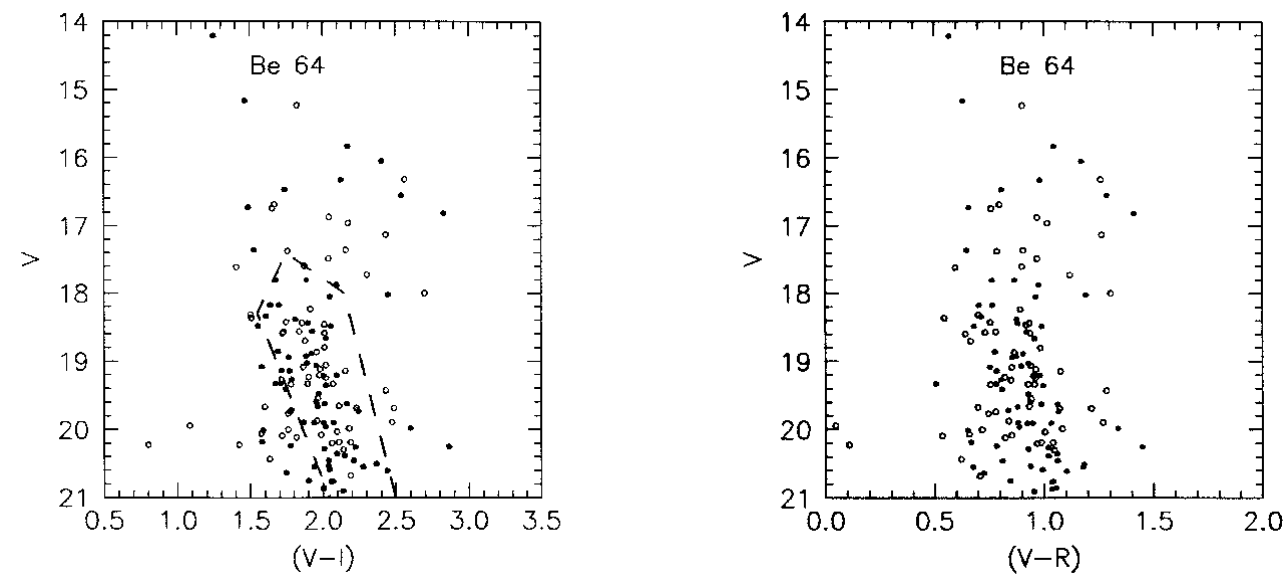

Fig. 6. The CMDs for two nearby fields towards east (open circles) and west (filled circles) directions at $30^{\prime}$ away from the cluster Be 64

$(V, V-I)$ CMDs of the cluster region and nearby fields indicates that in the range of $V=16-17,(V-R)=1.0-1.2$ and $(V-I)=2.0-2.3$, there are 7 stars in the cluster region, whereas, in the nearby fields there are 2 and 3 stars respectively. Since the CMDs of nearby fields covers an area which is double as compared to the cluster region, it is expected that at the most 2 field stars can contaminate the sample of the red giant clump. It is rather difficult to identify the MS in the CMDs, however, a well defined blue envelope of the MS can be easily identified in $(V, V-I)$ and $(V, V-R)$ CMDs. A comparison of the $(V, V-I)$ CMD of the cluster with the CMDs of nearby fields manifests that cluster region has large number of stars having $(V-I)<1.7$ mag. whereas there are very few stars in the CMD of nearby field regions having $(V-I)<1.7 \mathrm{mag}$. We have plotted a box using thick dashed line in the CMDs of nearby fields to indicate the main distribution of stars in the CMD. The same box is also plotted in the $(V, V-I)$ 

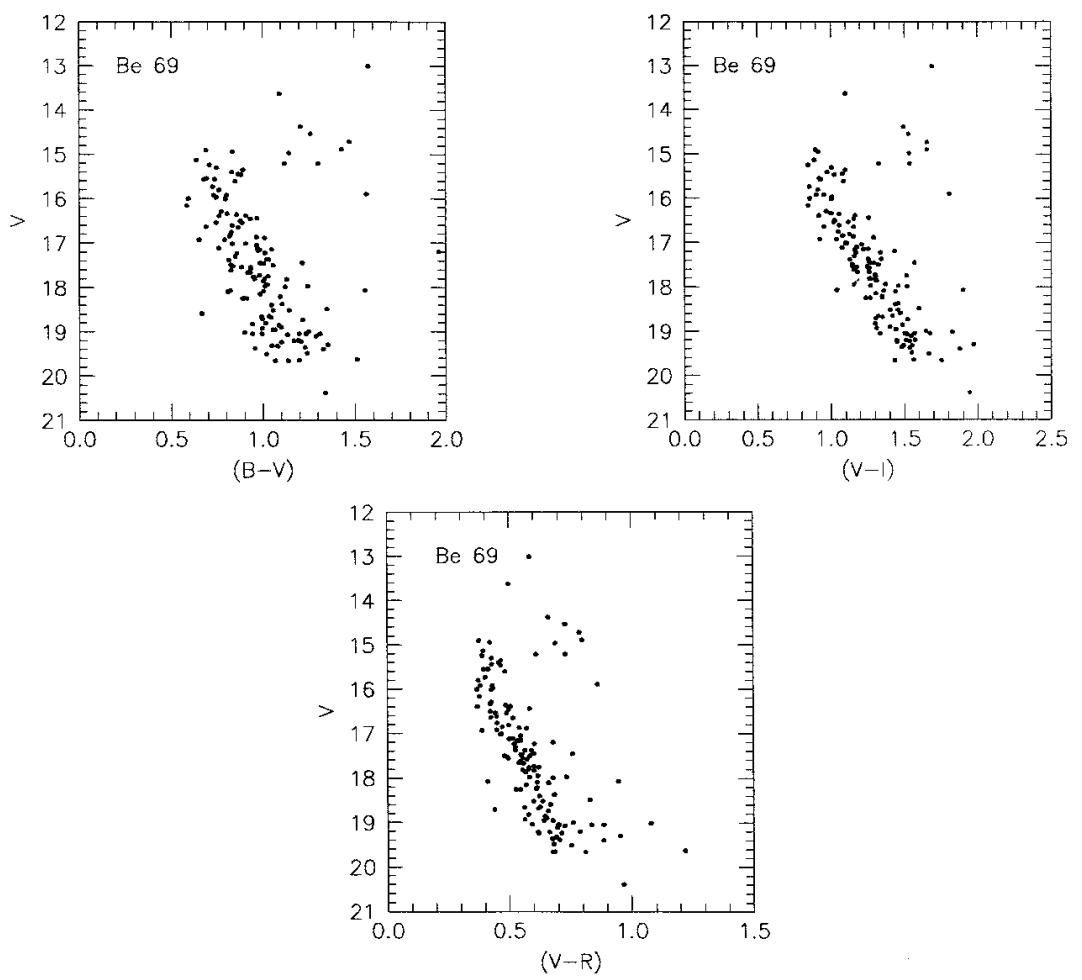

Fig. 7. The CMDs for all stars in Be 69 region
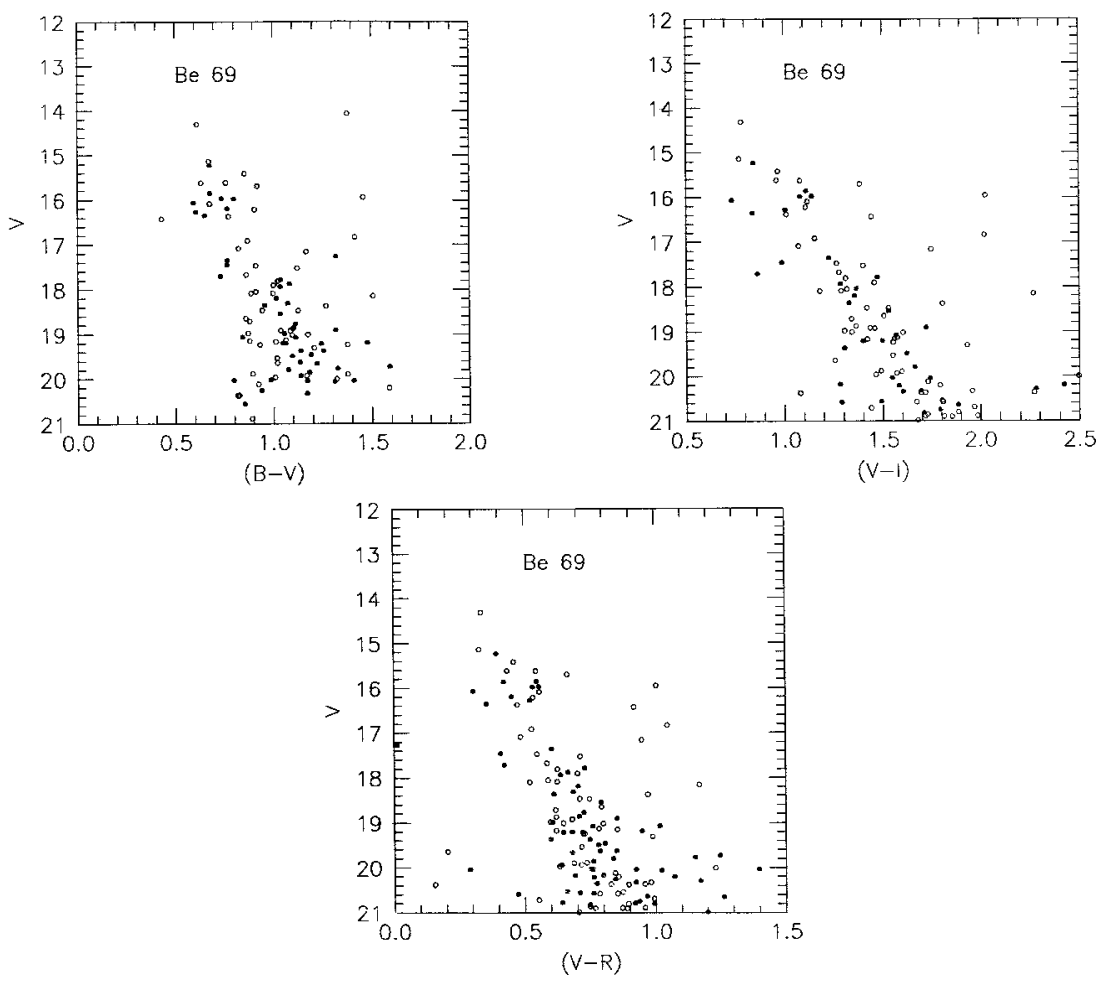

Fig. 8. The CMDs for two nearby fields towards north (open circles) and south (filled circles) directions at $30^{\prime}$ away from the cluster Be 69 
CMD of the cluster region. The stars in the cluster region lying in the box mainly belong to the foreground field population. The MS of the cluster region is negligibly affected by the field stars. Since the cluster is located at $b=4.6$, it indicates that the cluster may be located outside the galactic disk.

It is impossible to estimate the BTO and BRGB using $(V, B-V)$ CMD only, however, we have compared the morphology of the $(V, B-V),(V, V-I),(V, V-R)$ CMDs and estimated $V$ magnitude at the turnoff point on the $(V, V-I)$ and $(V, V-R)$ CMDs. Then $(B-V)$ colour corresponding to the so determined $V$ magnitudes was read from the $(V, B-V) \mathrm{CMD}$. The $(B-V)$ colour thus determined was taken as BTO. The BTO colour comes out to be $(B-V) \sim 1.05$. The position of BRGB is estimated at $V \approx 17.2$ and $(B-V) \approx 1.7$. The CIP comes out to be $\approx 0.65$ which indicates an age of $\sim 10^{9} \mathrm{yr}$.

\subsection{Be 69}

The CMDs for Be 69 are shown in Fig. 7. CMDs for two nearby fields towards north and south directions $\sim 30^{\prime}$ away from the cluster region have also been observed to estimate contamination of field stars in the cluster region. The CMDs for field regions are shown in Fig. 8. The area of the cluster region is $\sim 90 \%$ of the field region. Although the contamination due to field stars is significant, still the main properties of the cluster population can be seen in the CMD of the cluster region. The broadness of the main sequence is due to various causes, among which are the photometric errors, the presence of unresolved binary stars, a possible internal reddening and field stars contamination. The BTO is estimated at $V \sim 15.2$, $(B-V) \sim 0.75$, and position of BRGB is estimated at $V \sim 15.4,(B-V) \sim 1.30$. The colour index parameter comes out to 0.55 , which indicates an age of $10^{9} \mathrm{yr}$ for the cluster.

Here we would like to point out that the ages obtained by using this method are approximate. Error in eye estimate of CIP is approximately of the order of \pm 0.1 . For a value of CIP $=0.65 \pm 0.1$, age estimate can vary from $\log$ $t=8.7$ to $\log t=9.2$.

\section{Interstellar extinction}

To estimate interstellar extinction in the clusters, we have used $(U-B, B-V)$ colour diagram. Since a well defined blue envelope of the MS can be easily identified in the CMDs, we have considered only those stars as probable MS stars which lie between the blue envelope and a red envelope, obtained by shifting the MS by $\sim 0.3 \mathrm{mag}$ in $(B-V)$ colour. Probable main-sequence (MS) stars have been grouped in $(B-V)$ colour bins of 0.1 mag. The central value of each bin and the mean value $(U-B)$ is plotted in Figs. 9 and 10 for Be 64 and Be 69 respectively. The vertical bars are the standard errors. We find a mean reddening of $E(B-V)=1.05 \pm 0.05 \mathrm{mag}$ for Be 64 and $E(B-V)=0.65 \pm 0.05$ mag. for Be 69. The intrinsic ZAMS given by Mermilliod (1981) was used and the slope of $E(U-B) / E(B-V)$ was taken to be equal to 0.72 (Johnson \& Morgan 1953).

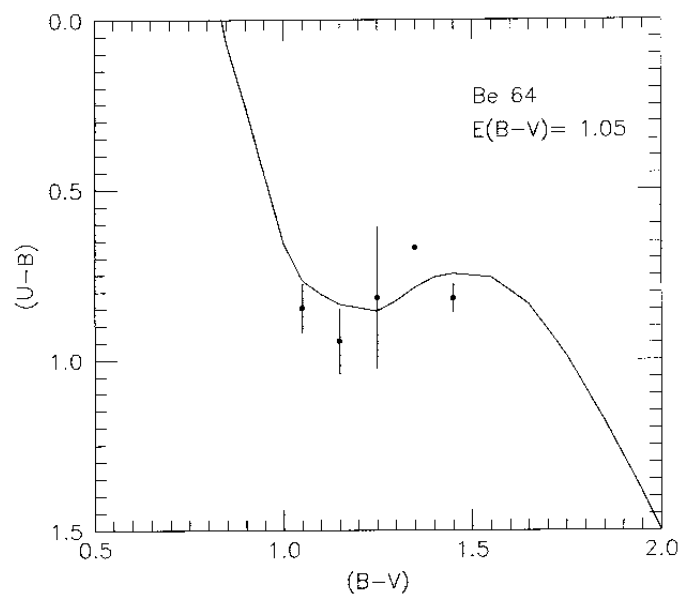

Fig. 9. The colour - colour diagram for the probable main sequence stars in the cluster Be 64

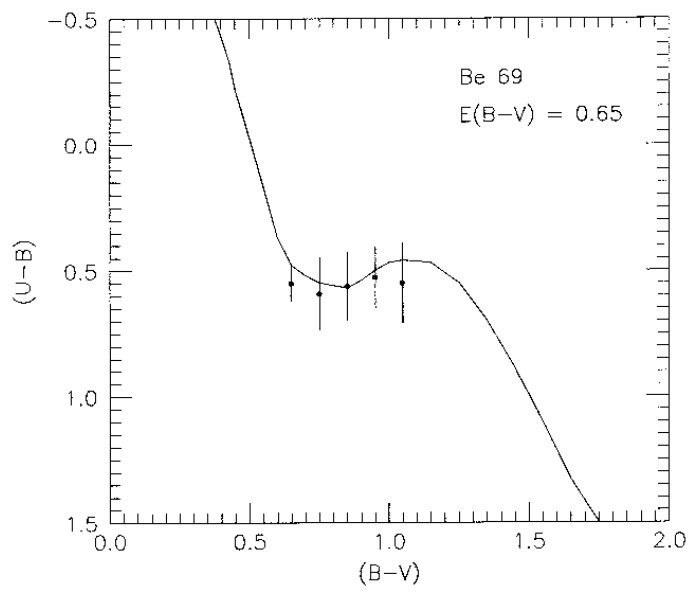

Fig. 10. The colour - colour diagram for the probable main sequence stars in the cluster Be 69

\section{Comparison of the CMDs with theoretical isochrones}

In the past few years the comparison of stellar evolutionary models with observations of old open clusters has become interesting because the CMD of old open clusters might be quite sensitive to the effects of convective overshoot mixing. Now it is possible to compare models computed without overshoot e.g., those by VandenBerg 

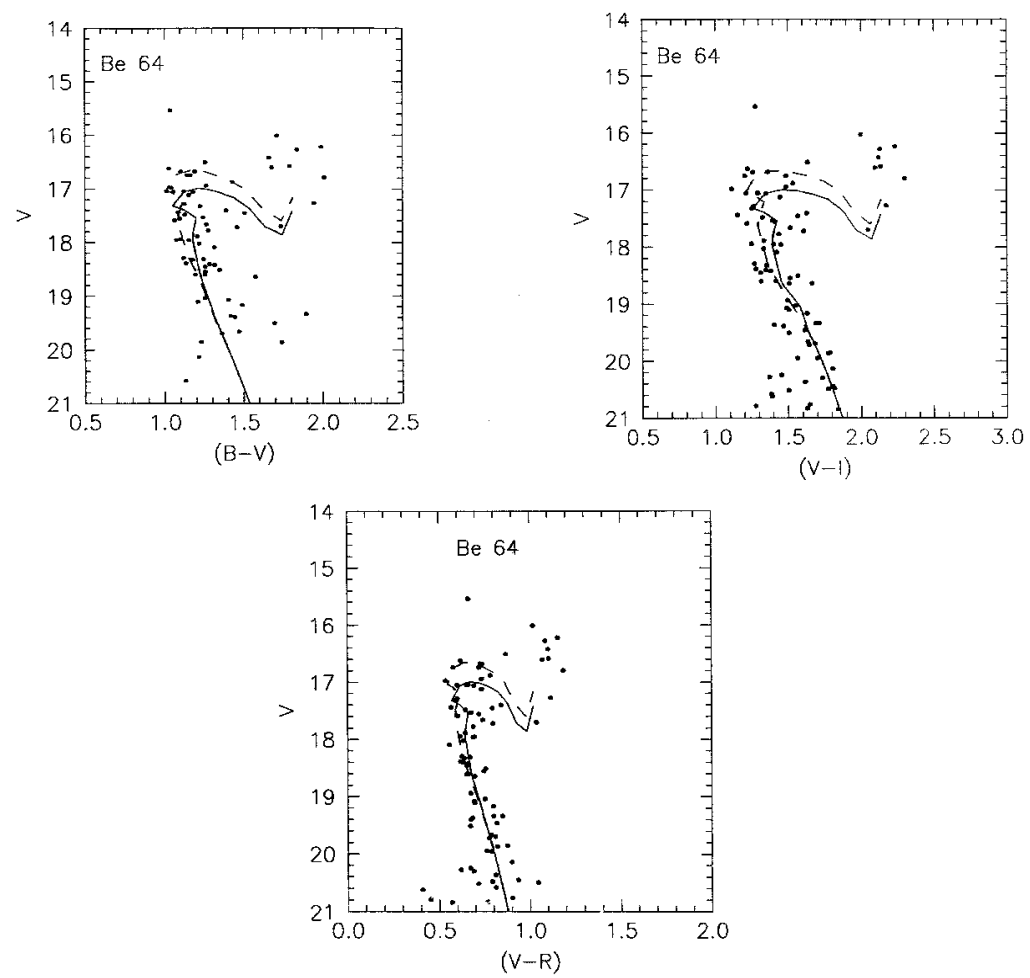

Fig. 11. The comparison of evolutionary model of VandenBerg (1985) with the CMDs of Be 64. The contribution of probable foreground stars have been removed

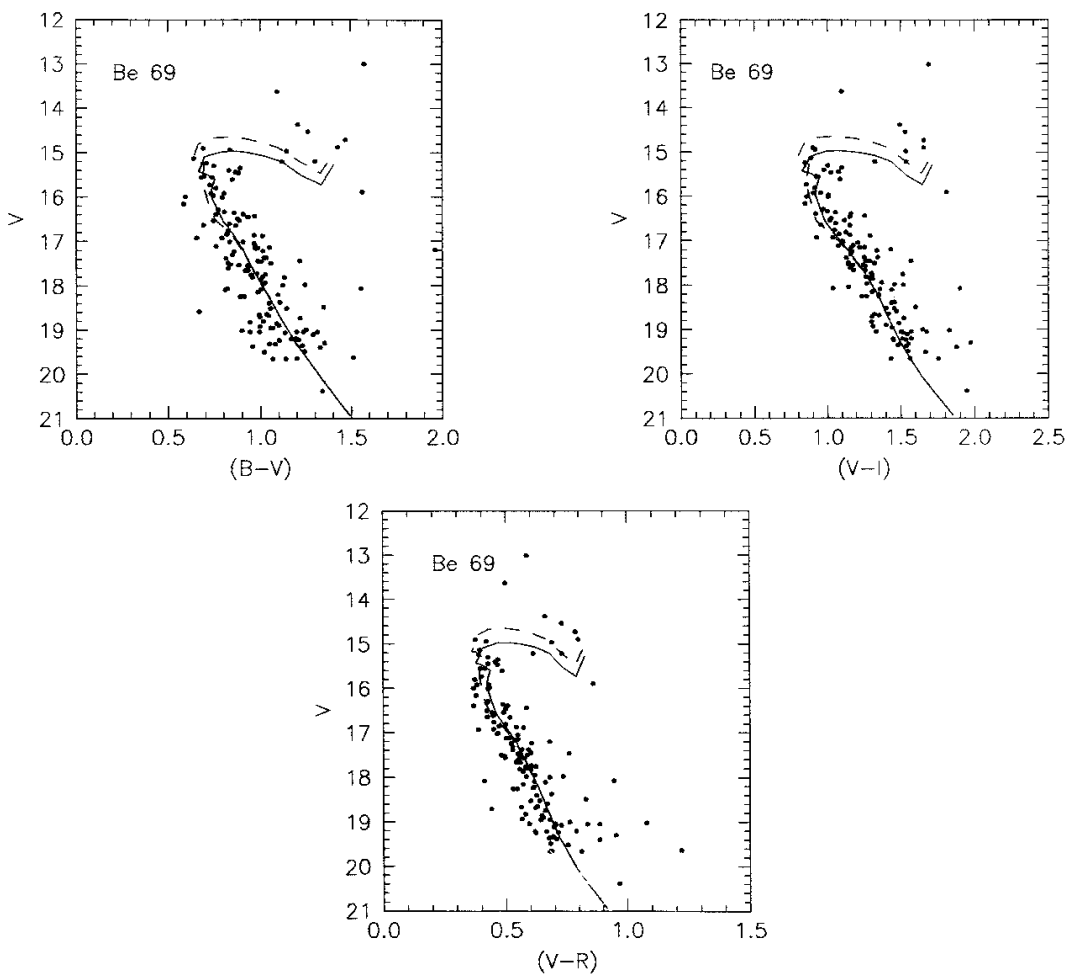

Fig. 12. Same as Fig. 11 but for Be 69 

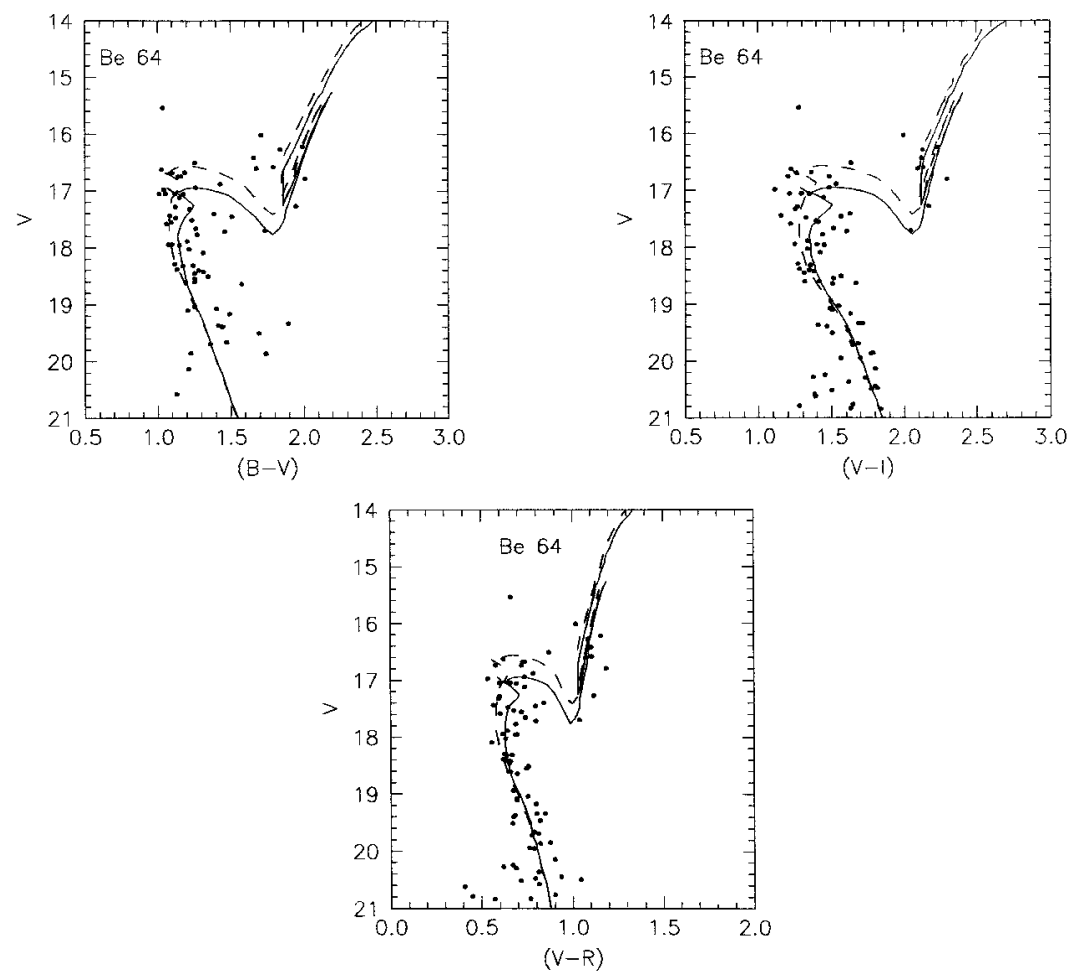

Fig. 13. The comparison of theoretical isochrones of Bertelli (1994) with the CMDs of Be 64
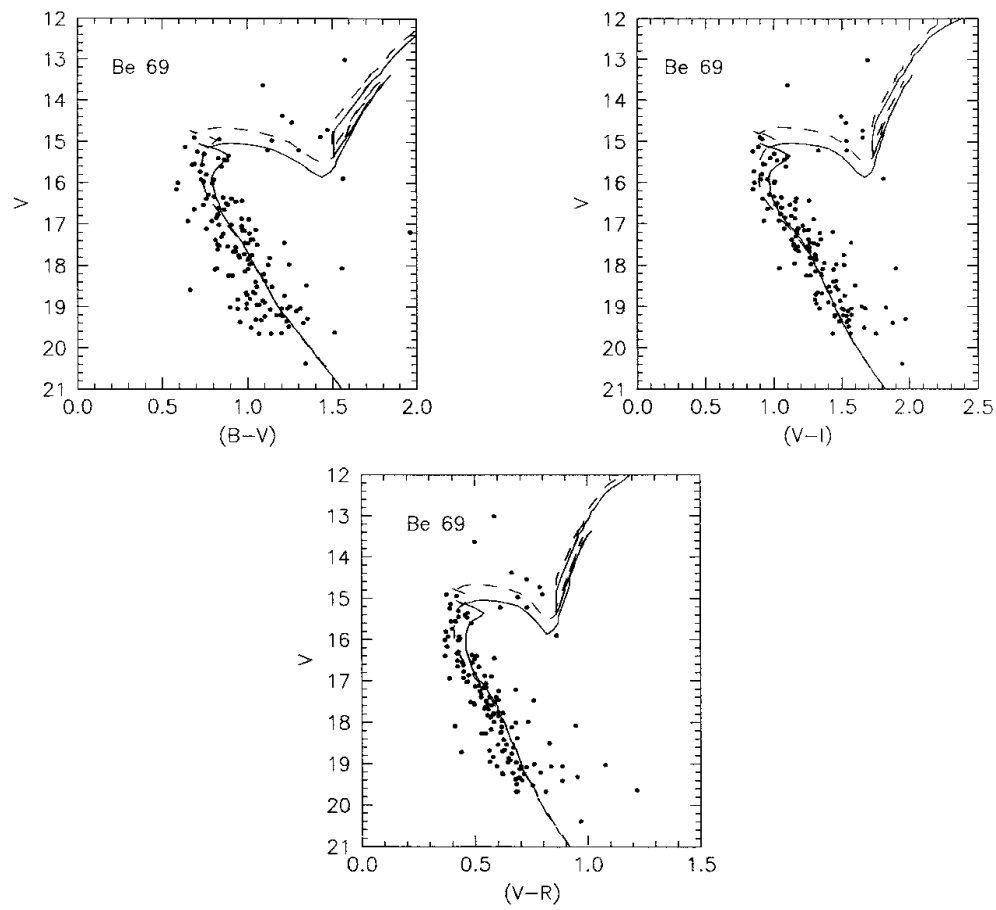

Fig. 14. Same as Fig. 13 but for Be 69 
(1985) with models incorporating overshoot (e.g., Bertelli et al. 1994). Age and distance of the cluster can also be obtained by fitting theoretical isochrones to the CMDs. The major problem in determining the age and distance modulus by comparing the theoretical isochrones with the observed CMDs, is the absence of data about the metal abundance $[\mathrm{Fe} / \mathrm{H}]$. Therefore we have used isochrones of different metallicity and tried to find out which provides the best global fit.

The clean CMDs of Be 64 after removing contribution of probable foreground stars have been used to compare with the theoretical isochrones. The best comparison with the standard evolutionary models of VandenBerg (1985), shown in Fig. 11, yields an age $\sim 0.8-1.0$ Gyr and $(m-M)=16.0$ for $Z=0.01$. The comparison in Fig. 11 is rather difficult to interpret because of scatter in the turnoff region. The comparison of CMDs of Be 69 with the standard models of VandenBerg (1985) is shown in Fig. 12. The best comparison yields an age of $\sim 0.8-1.0 \mathrm{Gyr}$ and $(m-M)=14.0$ for $Z=0.006$. However, in case of Be 69, there seems an apparent discrepancy between the shape of turnoff and the isochrones. This discrepancy has been noted before by several authors (e.g; Anthony-Twarog et al. 1991; Alfaro et al. 1992) and can be explained as a failure of the standard isochrones to include convective overshoot.

Theoretical isochrones with convective overshoot by Bertelli et al. (1994) have also been compared with the CMDs of Be 64 and Be 69 in Figs. 13 and 14. The best fit is obtained for $Z=0.008$, age $\sim 0.8-1.0 \mathrm{Gyr}$ for both the clusters. The apparent distance modulus $(m-M)$ comes out to be 16.2 (distance $=3880 \pm 480 \mathrm{pc}$ ) and 14.3 (distance $=2860 \pm 310 \mathrm{pc}$ ) for Be 64 and Be 69, respectively. The errors in distance estimates are the combination of errors in interstellar extinction and in visual fitting of the isochrones to the observations. The morphological features of the CMDs match very well with the theoretical isochrones predicted by the Bertelli et al. (1994). However, to discriminate between different evolutionary scenarios photometry with membership determination and spectroscopic determination of metallicity are required.

\section{Discussion and conclusions}

\subsection{Evolved stars in the cluster}

The spatial distribution of the evolved stars in Be 64 and Be 69, shown in Figs. 15 and 16, manifests that evolved stars have preferential distribution in the outskirts of the clusters. Hawarden (1975) has also found that giant branch clump stars in old open clusters are in general less centrally concentrated than the brightest stars remaining on the upper MS.

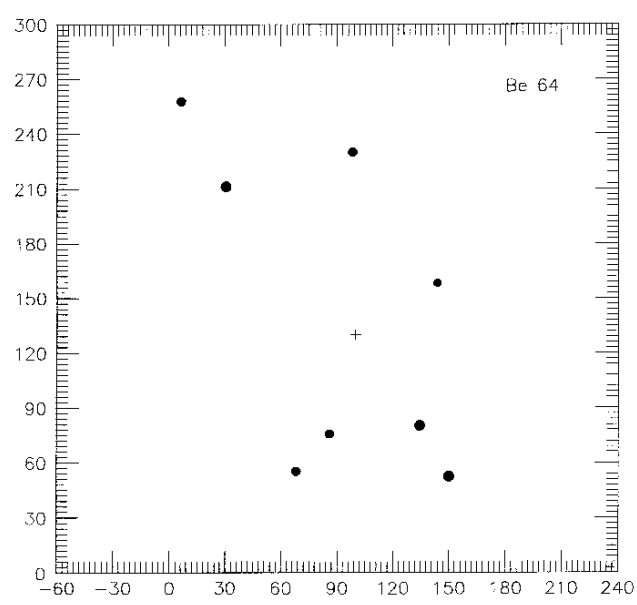

Fig. 15. The spatial distribution of the evolved stars in the cluster Be 64. The cross represents adopted center of the cluster

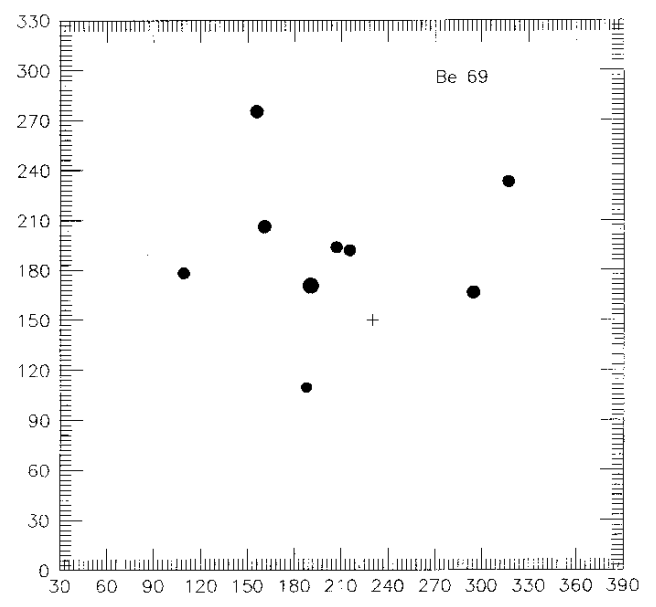

Fig. 16. Same as Fig. 15 but for Be 69

\subsection{Metallicity location relation}

Adopting a value of $R_{\odot}=8.5 \mathrm{Kpc}$, the galactocentric distance for the clusters has been calculated. The $[\mathrm{Fe} / \mathrm{H}]$ value has been obtained by using the relation $\log Z=0.977[\mathrm{Fe} / \mathrm{H}]-1.699$ from Bertelli et al. (1994). The various parameters obtained are given in Table 4 . The metallicity of these two clusters is in accordance with the radial gradient obtained by Friel \& Janes (1993) and Carraro \& Chiosi (1994).

The $z$ distance of Be $69(\sim 90$ pc below the formal galactic plane $)$ and Be $64(\sim 310 \mathrm{pc}$ above the formal galactic plane) suggests that Be 69 is situated in the anticenter direction of the thin disk whereas the cluster Be 64 may be located in the thick disk. The value of the metallicity for $\mathrm{Be} 64([\mathrm{Fe} / \mathrm{H}] \sim-0.4)$ is also close to the average value $([\mathrm{Fe} / \mathrm{H}] \sim-0.5)$ of the metallicity for the thick disk population (Sandage \& Fouts 1987). 


\subsection{Conclusions}

(1) We present photometry for two previously unstudied open clusters. Overall morphology of the CMDs of both clusters indicates that clusters are of intermediate age.

(2) The reddening for Be 64 and Be 69 comes out to be $E(B-V) \sim 1.05 \pm 0.05$ and $E(B-V) \sim 0.65 \pm$ 0.05. From mapping of interstellar reddening done by Pandey \& Mahra (1987), values of $E(B-V) \sim 1.10$ and $E(B-V) \sim 0.65$ have been obtained towards the Be 64 and Be 69 direction, respectively, which are in good agreement with the values obtained in the present study.

(3) The estimated metallicity for the clusters $(Z=0.008$, $[\mathrm{Fe} / \mathrm{H}] \sim-0.40)$, and the galactocentric distance of the clusters follow the radial gradient in metallicity obtained by Friel \& Janes (1993) and Carraro \& Chiosi (1994). The location of Be 69 puts the cluster in the thin disk towards the anticenter direction, whereas the location of Be 64 suggests that the cluster may be a member of the thick disk. (4) The evolved stars appear to be concentrated in the outskirts of the clusters.

Acknowledgements. This work is partly supported by the Department of Science and Technology (India) under the grant $\mathrm{SP} / \mathrm{S} 2 / \mathrm{O}-07 / 93$. Authors are thankful to the referee Dr. G. Carraro for useful comments.

\section{References}

Alfaro E.J., Aparicio A., Delgado A.J., Garcia-Pelayo J.M., Cabrera-Cano J., 1992, AJ 103, 204
Anthony-Twarog B.J., Heim E.A., Twarog B.A., Caldwell N., 1991, AJ 102, 1056

Bertelli G., Bressan A., Chiosi C., Fagotto F., Nasi E., 1994, A\&AS 106, 275

Carraro G., Chiosi C., 1994, A\&A 287, 761

Friel E.D., Janes K.A., 1993, A\&A 267, 75

Hawarden T.G., 1975, MNRAS 173, 223

Janes K.A., Adler D., 1982, ApJS 49, 425

Janes K.A., Phelps R., 1990, in: Philip A.G.D., Hayes D.S., Adelman S.J. (eds.) CCD in Astronomy II. L. Davis Press, Schenectady, p. 177

Johnson H.L., Morgan W.W., 1953, ApJ 117, 313

Kaluzny J., 1990, MNRAS 243, 492

Kaluzny J., 1994, A\&AS 108, 151

Landolt A.U., 1983, AJ 88, 439

Mermilliod J.C., 1981, A\&A 97, 235

Mohan V.M., Paliwal D.C., Mahra H.S., 1991, BASI 19, 235

Pandey A.K., Mahra H.S., 1987, MNRAS 226, 635

Pandey A.K., Bhatt B.C., Mahra H.S., 1987, Astrophys. Space Sci. 129, 293

Phelps R.L., Janes K.A., Montgomery K.A., 1994, AJ 107, 1079

Sandage A.R., Fouts G., 1987, AJ 93, 74

Spitzer L., 1958, ApJ 127, 17

Stetson P.B., 1987, PASP 99, 191

Twarog B.A., Anthony-Twarog B.J., McClure R.D., 1993, PASP 105, 78

VandenBerg D.A., 1985, ApJS 58, 711 
Table 1. Details of observation

\begin{tabular}{|c|c|c|c|c|}
\hline Date & Field & Filter & Exp.(sec) & No. of frames \\
\hline \multicolumn{5}{|l|}{$\overline{\text { Be } 64}$} \\
\hline \multirow[t]{2}{*}{21 oct-90 } & Cluster & $U$ & 3000 & 5 \\
\hline & Region & & & \\
\hline$" \prime$ & " & $B$ & 1500 & 3 \\
\hline " & " & V & 660 & 3 \\
\hline " & " & $R$ & 230 & 3 \\
\hline " & " & $I$ & 230 & 3 \\
\hline \multirow{2}{*}{${ }_{\prime \prime}^{10}$ Nov-90 } & " & $U$ & 2700 & 3 \\
\hline & " & $B$ & 1200 & 2 \\
\hline " & " & V & 600 & 2 \\
\hline " & " & $R$ & 300 & 2 \\
\hline " & " & $I$ & 300 & 2 \\
\hline \multirow{2}{*}{${ }_{\prime \prime}^{17}$ Nov-92 } & " & $U$ & 4200 & 3 \\
\hline & " & $B$ & 2100 & 2 \\
\hline "I & " & $V$ & 500 & 1 \\
\hline \multirow{2}{*}{${ }_{\prime \prime}^{18}$ Nov-92 } & " & $U$ & 3600 & 2 \\
\hline & " & $B$ & 1800 & 2 \\
\hline " & " & V & 300 & 1 \\
\hline "I & " & $R$ & 330 & 2 \\
\hline "I & " & $I$ & 230 & 2 \\
\hline \multirow{2}{*}{$\underset{\prime \prime}{24 \text { Nov-92 }}$} & " & $U$ & 5400 & 3 \\
\hline & " & $B$ & 2700 & 3 \\
\hline 18 Nov-92 & $\begin{array}{l}\text { Nearby } \\
\text { (East) }\end{array}$ & $V$ & 300 & 1 \\
\hline " & "I & $R$ & 320 & 2 \\
\hline " & " & $I$ & 320 & 2 \\
\hline 18 Nov-92 & $\begin{array}{l}\text { Nearby } \\
\text { (West) }\end{array}$ & V & 300 & 1 \\
\hline "I & il & $R$ & 320 & 2 \\
\hline " & " & $I$ & 320 & 2 \\
\hline \multicolumn{5}{|l|}{ Be 69} \\
\hline 22 oct-90 & $\begin{array}{l}\text { Cluster } \\
\text { (South) }\end{array}$ & $U$ & 3600 & 6 \\
\hline \multirow{2}{*}{$\begin{array}{l}\prime \prime \\
\prime \prime\end{array}$} & "I & $B$ & 2400 & 4 \\
\hline & " & $V$ & 900 & 3 \\
\hline \multirow{2}{*}{$\begin{array}{l}\prime \prime \\
\prime \prime\end{array}$} & " & $R$ & 300 & 2 \\
\hline & $\prime \prime$ & $R$ & 20 & 1 \\
\hline \multirow{2}{*}{$\begin{array}{l}\prime \prime \\
\prime \prime\end{array}$} & " & $I$ & 600 & 2 \\
\hline & " & $I$ & 20 & 1 \\
\hline \multirow{2}{*}{${ }_{\prime \prime}^{13}$ Nov-90 } & " & $U$ & 3600 & 4 \\
\hline & " & $B$ & 1200 & 2 \\
\hline 23 Nov-90 & $\begin{array}{l}\text { Cluster } \\
\text { (North) }\end{array}$ & $B$ & 600 & 1 \\
\hline$" \prime$ & $" \prime$ & $V$ & 1200 & 3 \\
\hline "I & " & $R$ & 300 & 2 \\
\hline " & " & $I$ & 450 & 2 \\
\hline 24 Nov-90 & " & $U$ & 2700 & 3 \\
\hline 1 & " & $B$ & 1800 & 2 \\
\hline 21 Dec-90 & " & $U$ & 3600 & 4 \\
\hline$" 1$ & $\prime \prime$ & $B$ & 1800 & 3 \\
\hline " & " & $V$ & 600 & 2 \\
\hline$\prime \prime$ & " & $R$ & 300 & 2 \\
\hline " & " & $I$ & 300 & 2 \\
\hline 22 Dec-92 & $\begin{array}{l}\text { Nearby } \\
\text { (North) }\end{array}$ & $B$ & 2700 & 3 \\
\hline " & $" 1$ & $V$ & 900 & 3 \\
\hline " & " & $V$ & 10 & 1 \\
\hline "I & " & $R$ & 450 & 3 \\
\hline " & " & $I$ & 450 & 3 \\
\hline 22 Dec-92 & $\begin{array}{l}\text { Nearby } \\
\text { (South) }\end{array}$ & $B$ & 2700 & 3 \\
\hline " & " & V & 200 & 2 \\
\hline " & " & $R$ & 400 & 2 \\
\hline "I & " & $I$ & 400 & 2 \\
\hline
\end{tabular}

Table 4. Galactic coordinates and estimated parameters of open clusters analysed in the paper

\begin{tabular}{lll}
\hline Cluster & Be 64 & Be 69 \\
\hline Parameter & & \\
\hline$l$ & 131.9 & 174.4 \\
$b$ & 4.6 & -1.8 \\
$E(B-V)$ & $1.05 \pm 0.05$ & $0.65 \pm 0.05$ \\
$E(B-V)^{*}$ & $\sim 1.10$ & $\sim 0.65$ \\
Distance, $d_{\odot}(\mathrm{Kpc})$ & $3.88 \pm 0.48$ & $2.86 \pm 0.31$ \\
Galactocentric & $11.46 \pm 0.40$ & $11.35 \pm 0.30$ \\
distance $(\mathrm{Kpc})$ & & \\
$z(\mathrm{pc})$ & $310 \pm 40$ & $-90 \pm 10$ \\
{$[\mathrm{Fe} / \mathrm{H}]$} & -0.40 & -0.40 \\
\hline
\end{tabular}

* Estimated from the work of Pandey \& Mahra (1987) see text (Discussion and conclusion). 